\title{
Electrical Characterization of Impurity-Free Disordered p-Type GaAs
}

\author{
Prakash N. K. Deenapanray, ${ }^{\mathrm{Z}}$ V. A. Coleman,* and C. Jagadish** \\ Department of Electronic Materials Engineering, Research School of Physical Sciences and Engineering, \\ The Australian National University, Canberra, Australian Capital Territory 0200, Australia
}

\begin{abstract}
Impurity-free disordered $\mathrm{p}-\mathrm{GaAs}$ epi layers by $\mathrm{SiO}_{2}$ or native oxide capping are characterized using deep level transient spectroscopy (DLTS) and capacitance-voltage measurements. Samples, including an uncapped epi layer for reference, were annealed at $900^{\circ} \mathrm{C}$ for $30 \mathrm{~s}$ under $\mathrm{Ar}$ ambient. Disordering resulted in an increase in the free hole concentration with the effect being more pronounced for the $\mathrm{SiO}_{2}$ capping layer. DLTS measurements revealed the corresponding increase in the concentrations of both the $\mathrm{Cu}$ and $\mathrm{Zn}$-related deep levels in the disordered epi layers. We relate these changes in electrical properties to segregation effects resulting from the nonequilibrium injection of excess gallium vacancies in the disordered p-type epi layers. We discuss the relative merit of using either $\mathrm{SiO}_{2}$ or native oxide layers for the integration of optoelectronic devices.

(C) 2003 The Electrochemical Society. [DOI: 10.1149/1.1543335] All rights reserved.
\end{abstract}

Manuscript received October 10, 2002. Available electronically January 10, 2003.

The integration of optoelectronic devices based on planar configurations may only be realized if suitable methods are available to modify the bandgap of III-V semiconductor heterostructures selectively. Two post-growth alternatives to the current, but relatively cumbersome, selective area epitaxy technique are $(i)$ impurity-free disordering (IFD), and (ii) implantation-induced disordering (IID). ${ }^{1-8}$ Although IID provides the better reproducibility, IFD may be the preferred option for many device applications because it is simple to implement, and, more importantly, it retains the better optical and electrical quality of the disordered semiconductor material. ${ }^{6,9}$ IFD can be achieved using a dielectric material such as $\mathrm{SiO}_{2}{ }^{1-8}$ or a native oxide ${ }^{6,8,10-12}$ layer in conjunction with high temperature annealing, whereas it can be suppressed by employing a capping layer (or stacking layer) having a larger thermal expansion coefficient than the III-V semiconductor. ${ }^{6,8,13,14}$ IFD using native oxide layers is highly desirable because these insulating layers also provide compatibility for the monolithic integration of optoelectronic devices. It is generally accepted that the disordering process is promoted by the creation and diffusion of gallium vacancies, $V_{\mathrm{Ga}}$, in GaAs. ${ }^{1-8,10-14}$ The creation of $V_{\mathrm{Ga}}$ is controlled by either the outdiffusion of $\mathrm{Ga}$ atoms into the capping layer or the time-dependent metallurgical reaction between GaAs and the encapsulant, $6,8,12-14$ while its diffusion away from the encapsulant/GaAs interface is stress controlled. ${ }^{5,8,13,14}$ Furthermore, we have recently proposed that the conversion of $V_{\mathrm{Ga}}$ into the arsenic-antisite, $\mathrm{As}_{\mathrm{Ga}}$ (i.e., $V_{\mathrm{Ga}}$ $+\mathrm{As}_{\mathrm{i}} \rightarrow \mathrm{As}_{\mathrm{Ga}}$ ) is enhanced when the near-surface region of GaAs is under compressive stress during annealing. ${ }^{15}$

Despite the apparent simplicity of IFD and its two decades of research, the operative mechanisms underlying the disordering process still raise open-ended questions. This is largely due to the fact that disordering is the compound effect of several critical factors, such as the quality of the capping layer, annealing conditions, stress, and doping. ${ }^{16}$ Furthermore, most of the past studies of IFD have excluded the defect engineering aspects of the process, including the defect-driven atomic relocation processes. Due to growth and dopant diffusion issues, most optoelectronic devices contain a heavily doped p-type ( $\mathrm{Zn}$ or $\mathrm{Be}$ ) top layer. Since $\mathrm{Zn}$ or Be are fast diffusers, the injection of $V_{\mathrm{Ga}}$ during disordering of the heavily doped top layer may result in their redistribution. Haddara et al. ${ }^{17}$ have demonstrated the atomic relocation of a Be marker in GaAs grown by molecular beam epitaxy using $\mathrm{SiO}_{2}$ and $\mathrm{Si}_{3} \mathrm{~N}_{4}$ capping layers. The long-range diffusion of these dopants lead to the unwanted, spatially nonselective impurity-induced disordering of the III-V heterostructures (i.e., poor electrical and optical properties of devices). ${ }^{18-20}$ Pre-

\footnotetext{
* Electrochemical Society Student Member.

** Electrochemical Society Active Member.

${ }^{\mathrm{z}}$ E-mail: pnk109@rsphysse.anu.edu.au
}

vious reports have studied the contentious issue of dopant diffusion under equilibrium conditions, which are not applicable under the conditions required to achieve IFD. ${ }^{18-20}$ Furthermore, it is of technological significance to research dielectric capping layers that can promote IFD while minimizing the redistribution of the fastdiffusing dopants.

In this paper, we compare the segregation of impurities, such as $\mathrm{Zn}$ and $\mathrm{Cu}$, under nonequilibrium annealing conditions in p-type GaAs capped with either a $\mathrm{SiO}_{2}$ or a native oxide layer. The redistribution of these species is discussed in light of the creation of $V_{\mathrm{Ga}}$ during the disordering process. Our results demonstrate that the disordering-induced relocation of $\mathrm{Zn}$ is less pronounced when a native oxide layer is used. Hence, native oxide layers may be more suitable for IFD of GaAs-based heterostructures containing a p-doped top layer.

\section{Experimental}

p-Type $\operatorname{GaAs}(100)$ layers doped with $7 \times 10^{15} \mathrm{Zn} / \mathrm{cm}^{3}$ were grown at $750^{\circ} \mathrm{C}$ on $\mathrm{p}^{+}$-substrates by metallorganic chemical vapor deposition (MOCVD). A native oxide layer $\sim 30 \mathrm{~nm}$ thick was formed on one set of samples (area $4.5 \times 4.5 \mathrm{~mm}^{2}$ ) by pulsed anodic oxidation (ANO) at room temperature (period of rectangular current waveform $=20 \mathrm{~ms}$, duty cycle $=$ one-fourth, potential difference between electrodes $=40 \mathrm{~V}$ ). Details of our electrochemical cell have been described elsewhere. ${ }^{21}$ Thin native oxide layers (30 $\mathrm{nm}$ ) have recently been used successfully to engineer the bandgap of GaAs-based laser diodes for the fabrication of multiwavelength lasers by the IFD process. ${ }^{22}$ Another set of samples was capped with $30 \mathrm{~nm} \mathrm{SiO}_{2}$ by plasma-enhanced chemical vapor deposition. The capped samples, together with an as-grown layer, were annealed at $900^{\circ} \mathrm{C}$ for $30 \mathrm{~s}$ in $\mathrm{Ar}$ ambient by rapid thermal annealing (RTA). Titanium Schottky barrier diodes (SBDs), $200 \mathrm{~nm}$ thick and of 0.77 $\mathrm{mm}$ diam, were formed on the chemically cleaned layers by electron beam evaporation. SBDs were also fabricated on the as-grown epi layer. An In-Ga eutectic was used to form ohmic contacts on the back side of samples. The electrical properties of samples were measured by high-frequency $(1 \mathrm{MHz})$ capacitance-voltage $(\mathrm{C}-\mathrm{V})$ and deep level transient spectroscopy (DLTS) techniques. The room temperature $\mathrm{C}-\mathrm{V}$ measurements were used to determine the free hole concentration, $N_{\mathrm{A}}$, of the samples. The activation energy, $E_{\mathrm{t}}$, and apparent capture cross section, $\sigma_{\mathrm{a}}$, (i.e., "signature") of a defect level was extracted from the Arrhenius plot of $\ln \left(T^{2} / e_{\mathrm{h}}\right) v s .1000 / T$, where $e_{\mathrm{h}}$ is the hole emission rate and $T$ is the measurement temperature. Isothermal DLTS measurements were performed using fixed reverse bias and variable filling pulse heights to determine defect depth profiles. 


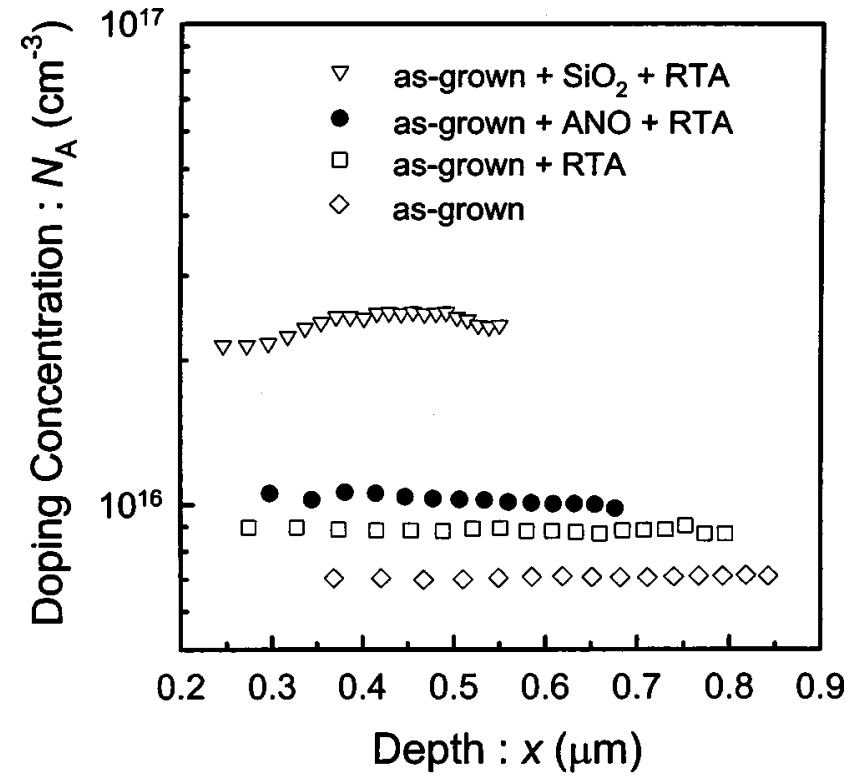

Figure 1. Free carrier concentration of as-grown (open diamonds) and annealed (capped or uncapped) samples. The legends indicate the annealing conditions. Samples were annealed in a rapid thermal annealer at $900^{\circ} \mathrm{C}$ for $30 \mathrm{~s}$ in an Ar ambient.

\section{Results and Discussion}

Figure 1 depicts the free carrier concentration, $N_{\mathrm{A}}$, in the asgrown (open diamonds) and annealed samples. The legends show the conditions under which the samples were annealed. The hole concentration in the as-grown layer is $7 \times 10^{15} \mathrm{~cm}^{-3}$, and it increases to $\sim 9 \times 10^{15} \mathrm{~cm}^{-3}$ upon RTA. This increase could result from the electrical activation of $\mathrm{Zn}$ which was otherwise passivated by hydrogen or occupied interstitial sites in the as-grown material. Additionally, annealing could increase the concentration of acceptor-type defects. The capped and annealed samples show further increase in $N_{\mathrm{A}}$ in the near-surface region with the effect being more pronounced for the $\mathrm{SiO}_{2}$-capped sample (open triangles). The annealed layers capped with ANO (solid circles) or $\mathrm{SiO}_{2}$ exhibit increases in $N_{\mathrm{A}}$ by $\sim 1.5$ or $\sim 3.8$ times, respectively, compared to the as-grown sample. The results in Fig. 1 clearly show that IFD increased the free hole concentration in the near-surface region of the p-type GaAs layer.

In order to understand the mechanism responsible for the change in $N_{\mathrm{A}}$ following IFD of p-GaAs, we turn to the DLTS spectra measured from the same samples. Figure 2 depicts the DLTS spectra measured from as-grown, as-grown and annealed, $\mathrm{SiO}_{2}$-capped and annealed, and ANO-capped and annealed p-type GaAs. The Arrhenius plots shown in open symbols in Fig. 3 were used to determine the signature of defects, while the solid lines overlaying the data points are the signatures of defects previously reported in the literature. ${ }^{23-25}$ This comparative analysis is a commonly used procedure for the identification of defects measured by DLTS. HB2 and $\mathrm{HC} 1$, which are commonly observed in $\mathrm{p}-\mathrm{GaAs}$ epilayers grown by MOCVD can be identified as $\mathrm{Cu}_{\mathrm{Ga}}$ (HL4) and Fe-related (HL3) defects, respectively. ${ }^{23}$ The DLTS depth profiles shown in Fig. 4a and $b$ (solid lines) reveal that the peak concentrations of $\mathrm{HC1}$ and $\mathrm{HB} 2$ in the as-grown layer were $\sim 2 \times 10^{13}$ and $\sim 3$ $\times 10^{13} \mathrm{~cm}^{-3}$, respectively. The decrease in the concentrations of $\mathrm{HC} 1$ and $\mathrm{HB} 2$ toward the surface indicates that $\mathrm{Fe}$ and $\mathrm{Cu}$ diffused from the heavily doped substrate into the epi layer during growth. Additional defect peaks $\mathrm{HA}, \mathrm{HB} 1$, and $\mathrm{HC} 2$ are detected in the uncapped and annealed layer. HC2 may appear to be similar to $\mathrm{HC} 1$, but for their different signatures (Fig. 3). Indeed, the peak position of $\mathrm{HC} 2$ is shifted to the lower temperature by $\sim 5 \mathrm{~K}$ compared to

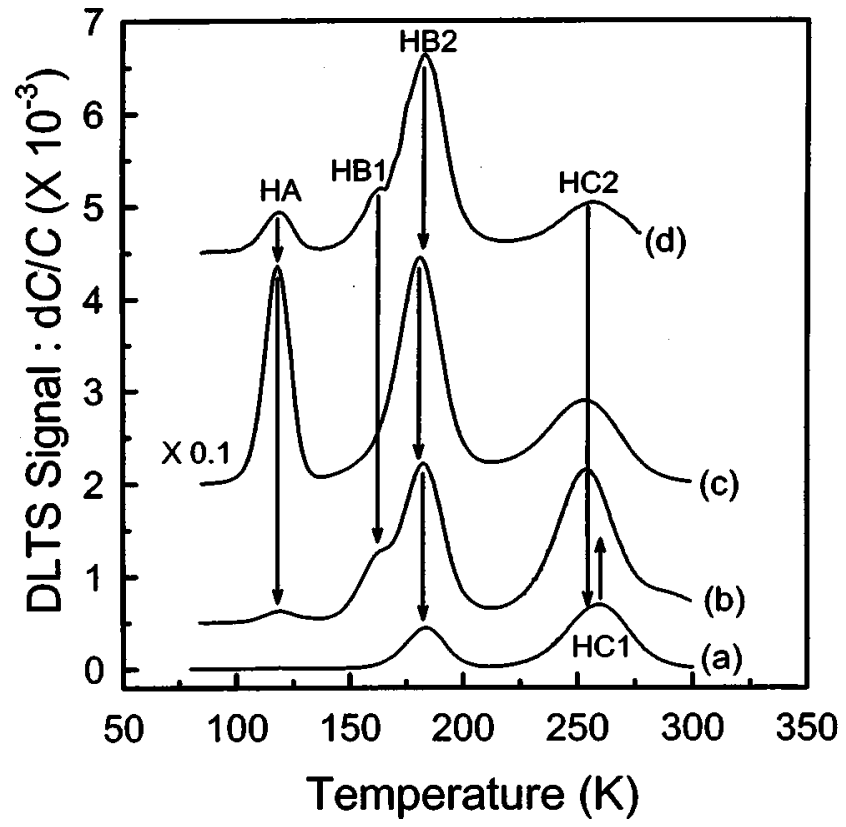

Figure 2. DLTS spectra measured from (a) as-grown (b) uncapped and annealed, (c) $\mathrm{SiO}_{2}$-capped, and (d) ANO-capped annealed p-type GaAs layers. Defects $\mathrm{HC} 1$ and $\mathrm{HB} 2$ are detected in the as-grown sample, while additional hole traps $\mathrm{HA}, \mathrm{HB} 1$, and $\mathrm{HC} 2$ are observed in the annealed samples.

that of HC1. HA can be identified as the Zn-related defect HL12 that has previously been reported in $\mathrm{Zn}$-contaminated material grown by liquid phase epitaxy, ${ }^{23}$ while $\mathrm{HB} 1$ may be similar to defect $\mathrm{H} 2$ ' that has been proposed to be a defect complex involving $\mathrm{Cu}$ and the arsenic interstitial, $\mathrm{Cu}-\mathrm{As}_{\mathrm{i}} .{ }^{24}$ The summary of the electrical properties of hole traps and their tentative identification is given in Table I. Figure 4 shows that the concentrations of all defects were increased following annealing of the as-grown sample. The detection of HA in the uncapped and annealed sample suggests the activation of $\mathrm{Zn}$ related defects which were electrically passivated by hydrogen in

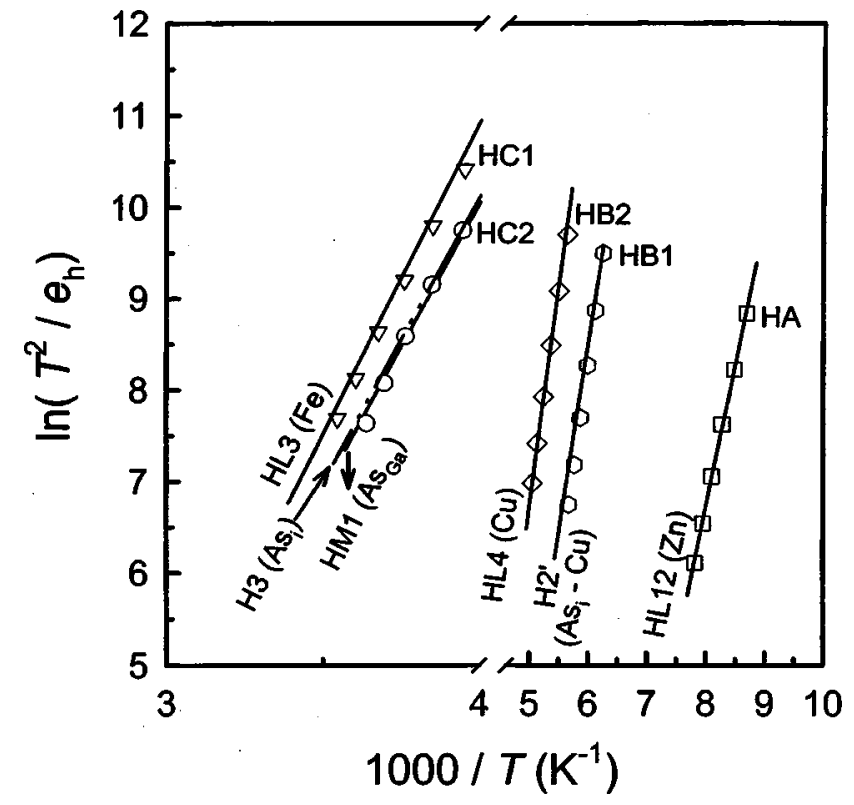

Figure 3. Arrhenius plots from which the signatures of defects are extracted. The solid lines overlaying the experimental data points (open symbols) are signatures of defects in $\mathrm{p}-\mathrm{GaAs}$ layers previously reported in the literature. 


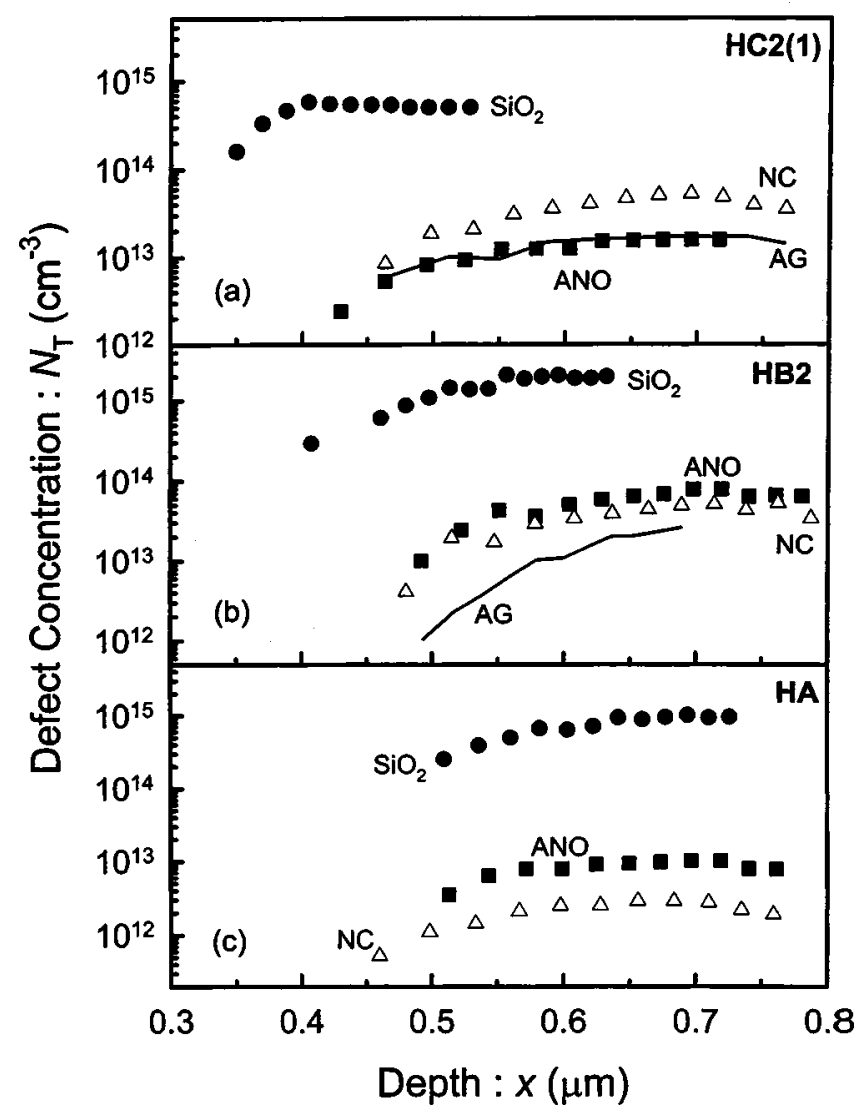

Figure 4. DLTS depth profiles of (a) HC2 and HC1, (b) HB2, and (c) HA. The solid circles, solid squares, open triangles, and solid lines correspond to $\mathrm{SiO}_{2}$-capped, ANO-capped, uncapped (NC), and as-grown (AG) samples, respectively. Defect HA is not detected in the as-grown sample. The depth profile of HB1 was not measured since its peak is either partially of completely masked by the larger peak of defect HB2.

the as-grown sample. HB2 is a p-type dopant in GaAs since it can be identified as the $\mathrm{Cu}_{\mathrm{Ga}}$ double acceptor state. ${ }^{23,25}$ Because its concentration does not increase significantly upon annealing (Fig. 4b), we may conclude that the increase in $N_{\mathrm{A}}$ in the uncapped and annealed sample (see Fig. 1) is mainly due to the electrical activation of $\mathrm{Zn}$.

Of greater significance here is the comparison of defects created in the capped and annealed samples. Figure 2 (spectrum (c)) and Fig. 4 clearly demonstrate that the concentrations of HA, HB2, and $\mathrm{HC} 2$ are increased by 2-3 orders of magnitude when a $\mathrm{SiO}_{2}$ capping layer is used. Judging from the asymmetric DLTS peak of HB2, we believe that $\mathrm{HB} 1$ is still present in the sample disordered by $\mathrm{SiO}_{2}$ capping. In contrast, the concentration of defects in p-GaAs disordered using a native oxide layer is $\sim 2$ orders of magnitude lower compared to a $\mathrm{SiO}_{2}$ layer (spectrum d in Fig. 2 and Fig. 4). It is

Table I. Summary of the electrical properties of hole traps and their tentative identification.

\begin{tabular}{|c|c|c|c|}
\hline Defect & $\begin{array}{l}\text { Activation } \\
\text { energy, } E_{\mathrm{t}} \\
\quad(\mathrm{eV})\end{array}$ & $\begin{array}{l}\text { Capture } \\
\text { cross section, } \sigma_{\mathrm{a}} \\
\left(\mathrm{cm}^{2}\right)\end{array}$ & Identification \\
\hline HA & $0.27 \pm 0.02$ & $7.3 \times 10^{-14}$ & HL12 (Zn-related $)^{23}$ \\
\hline HB1 & $0.41 \pm 0.04$ & $5.0 \times 10^{-13}$ & $\mathrm{H} 2^{\prime}\left(\mathrm{Cu}-\mathrm{As}_{\mathrm{i}}\right)^{24}$ \\
\hline HB2 & $0.39 \pm 0.02$ & $6.3 \times 10^{-15}$ & HL4 (Cu-related $)^{23,24}$ \\
\hline $\mathrm{HC} 1$ & $0.58 \pm 0.03$ & $8.2 \times 10^{-15}$ & HL3 (Fe-related) $)^{23,24}$ \\
\hline $\mathrm{HC} 2$ & $0.56 \pm 0.03$ & $3.0 \times 10^{-15}$ & $\begin{array}{c}\mathrm{HM} 1\left(\mathrm{As}_{\mathrm{Ga}}\right) ?^{26 / \mathrm{H} 3} \\
\left(\mathrm{As}_{\mathrm{i}}\right) ?^{24}\end{array}$ \\
\hline
\end{tabular}

timely here to discuss the possible origin of HC2. Figure 3 shows that its signature is similar to those of defects $\mathrm{H}^{24}$ and HM1 ${ }^{26}$ In their study of electron irradiated epitaxially-grown p-GaAs, Bourgoin $e t a l{ }^{24}$ proposed that $\mathrm{H} 3$ was related to the arsenic interstitial, $\mathrm{As}_{\mathrm{i}}$, whereas Lagowski et al. ${ }^{26}$ provided the unambiguous evidence that MH1 detected in bulk material was the double donor state of EL2, i.e., $\mathrm{As}_{\mathrm{Ga}}(+/++)$. During $\mathrm{SiO}_{2}$-capped annealing, $\mathrm{Ga}$ atoms outdiffuse into the capping layer creating an excess of As over $\mathrm{Ga}$ in the near-surface region of the GaAs layer. Hence, the defects created in IFD GaAs are either $V_{\mathrm{Ga}}, \mathrm{As}_{\mathrm{i}}, \mathrm{As}_{\mathrm{Ga}}$, or complexes thereof as discussed in our recent studies on IFD of n-type GaAs. ${ }^{15,27}$ These defects are also created in $\mathrm{n}-\mathrm{GaAs}$ disordered using a native oxide layer. ${ }^{28}$ In this case, defects are created by the metallurgical reaction between the native oxide layer and GaAs. ${ }^{12,21}$ We have shown that the single donor state $(0 /+)$ of EL2 in disordered n-type GaAs exhibits a depth profile that decays exponentially below the surface. ${ }^{27}$ Figure $4 \mathrm{a}$ reveals that the depth profile of $\mathrm{HC} 2$ shows a conspicuous decrease toward the surface. Given that the hole trap related to EL2 has not been reported in epitaxially grown $\mathrm{p}-\mathrm{GaAs}$ to date and the depth profile of $\mathrm{HC} 2$, we suspect that HC2 is not the EL2. We defer any further discussion of $\mathrm{HC} 2$ since its identity is not known to us at present.

$\mathrm{Zn}$ and $\mathrm{Cu}$ diffuse by the same mechanisms, namely the kick-out (e.g., $\mathrm{Zn}_{\mathrm{i}}+\mathrm{Ga}_{\mathrm{Ga}} \rightarrow \mathrm{Zn}_{\mathrm{Ga}}+\mathrm{Ga}_{\mathrm{i}}$ ) or the substitutional (e.g., $\mathrm{Zn}_{\mathrm{i}}$ $+V_{\mathrm{Ga}} \rightarrow \mathrm{Zn}_{\mathrm{Ga}}$ ) process. ${ }^{17-20,25}$ Both mechanisms rely on the mobility of the interstitial species $\left(\mathrm{Zn}_{\mathrm{i}}\right.$ or $\left.\mathrm{Cu}_{\mathrm{i}}\right)$, and it is generally accepted that diffusion is predominantly by the kick-out mechanism. However, Haddara et al. ${ }^{17}$ have demonstrated that this picture cannot be maintained under the nonequilibrium injection of $V_{\mathrm{Ga}}$ (i.e., the IFD process) when the substitutional mechanism dominates. Hence, we may conclude that the creation of HA, HB1, and HB2 in the disordered $\mathrm{p}$-GaAs layers results from the segregation of $\mathrm{Zn}$ and $\mathrm{Cu}$ toward the surface of the samples. It is worth noting here that $\mathrm{HA}$ is most probably a complex involving $\mathrm{Zn}_{\mathrm{i}}$, since $\mathrm{Zn}_{\mathrm{Ga}}$ is a shallow acceptor. Since the concentration of $\mathrm{HB} 2\left(\mathrm{Cu}_{\mathrm{Ga}}\right)$ is relatively small in the sample disordered using $\mathrm{SiO}_{2}$ (Fig. 4b), we conclude that the increase in $N_{\mathrm{A}}$ in this sample (Fig. 1) is predominantly due to the segregation of $\mathrm{Zn}$. This is evidenced by the rather large concentration of HA in the sample (spectrum c) in Fig. 2 and Fig. 4c). The relatively smaller increase in $N_{\mathrm{A}}$ in the sample disordered using a native oxide layer agrees with the correspondingly lower segregation of impurities in the sample. The decrease in the concentration of defects toward the surface could be the result of the efficient conversion of $V_{\mathrm{Ga}}$ into $\mathrm{As}_{\mathrm{Ga}}$ in the near-surface region of $\mathrm{GaAs}$ which is under compressive stress during IFD. ${ }^{15}$

Most research in the past has studied IFD in nominally undoped structures using $\mathrm{SiO}_{2}$ capping layers. However, these results cannot be automatically transferred to doped device structures because of atomic relocation of $\mathrm{Zn}$ or Be during IFD. ${ }^{16,17,29}$ Two alternatives exist to this problem: $(i)$ using $\mathrm{C}$ as a p-type dopant, ${ }^{22}$ and/or (ii) employing a capping layer that is able to promote disordering while minimizing the redistribution of $\mathrm{Zn}$ or Be. Native oxide layers have been shown to effectively provide sufficient disordering in GaAsbased heterostructures for the fabrication of optoelectronic devices. ${ }^{10-12,22}$ The results discussed above clearly demonstrate that the segregation of impurities, especially $\mathrm{Zn}$, during the injection of $V_{\mathrm{Ga}}$ can be minimized by the use of a native oxide layer. Indeed, our preliminary results (to be presented elsewhere) on the bandgapengineered laser structures containing a heavily $\mathrm{Zn}$-doped top layer by IFD using a native oxide layer are very promising.

\section{Conclusion}

We have characterized impurity-free disordered p-GaAs layers using either a $\mathrm{SiO}_{2}$ or native oxide layer by DLTS and C-V techniques. Disordering causes the segregation impurities such as, $\mathrm{Zn}$ and $\mathrm{Cu}$, in the near-surface region of samples. We have discussed this atomic relocation process by the diffusion of these species by the substitutional mechanism under the nonequilibrium injection of 
$V_{\mathrm{Ga}}$. Further, our results have shown that the segregation of $\mathrm{Zn}$ is minimized when a native oxide layer is used for disordering, and offers new prospects for its use in the bandgap modification of optoelectronic devices that use $\mathrm{Zn}$ as a p-type dopant.

\section{Acknowledgment}

P.N.K.D is grateful for the financial support of the Australian Research Council.

\section{References}

1. D. G. Deppe, L. J. Guido, N. Holonyak, Jr., K. C. Hsieh, R. D. Burnham, R. L. Thornton, and T. L. Paoli, Appl. Phys. Lett., 49, 510 (1986).

2. P. N. K. Deenapanray, L. Fu, H. H. Tan, and C. Jagadish, Electrochem. Solid-State Lett., 3, 196 (2000); L. Fu, P. N. K. Deenapanray, H. H. Tan, C. Jagadish, L. V. Dao, and M. Gal, Appl. Phys. Lett., 76, 837 (2000).

3. S. Bürkner, M. Maier, E. C. Larkins, W. Rothemund, E. P. O'Reilly, and J. D. Ralston, J. Electron. Mater., 24, 805 (1995).

4. P. N. K. Deenapanray, H. H. Tan, M. I. Cohen, K. Gaff, M. Petravic, and C. Jagadish, J. Electrochem. Soc., 147, 1950 (2000); P. N. K. Deenapanray and C. Jagadish, J. Vac. Sci. Technol. B, 19, 1962 (2001).

5. P. N. K. Deenapanray and C. Jagadish, Electrochem. Solid-State Lett., 4, G11 (2001).

6. J. H. Marsh, Semicond. Sci. Technol., 8, 1136 (1993),

7. J. H. Teng, S. J. Chua, G. Li, A. S. Helmy, and J. H. Marsh, Appl. Phys. Lett., 76, 1582 (2000).

8. See, for example, Semiconductor Quantum Wells Intermixing, Optoelectronic Properties of Semiconductors and Superlattices, Vol. 8, E. H. Li, Editor, Gordon and Breach, Amsterdam (2000).

9. P. N. K. Deenapanray, H. H. Tan, B. G. Svensson, and C. Jagadish, Jpn. J. Appl. Phys., Part 1, 42, (unpublished) (2003).

10. S. Yuan, Y. Kim, C. Jagadish, P. T. Burke, M. Gal, J. Zou, D. Q. Cai, D. J. H. Cockayne, and R. M. Cohen, Appl. Phys. Lett., 70, 1269 (1997).

11. S. Yuan, Y. Kim, H. H. Tan, C. Jagadish, P. T. Burke, L. V. Dao, M. Gal, J. Zou, D.
Q. Cai, D. J. H. Cockayne, and R. M. Cohen, J. Appl. Phys., 83, 1305 (1998).

12. R. M. Cohen, G. Li, C. Jagadish, P. T. Burke, and M. Gal, Appl. Phys. Lett., 73, 803 (1998).

13. A. Pépin, C. Vieu, M. Schneider, H. Launois, and Y. Nissim, J. Vac. Sci. Technol. $B, 15,142$ (1997).

14. L. Fu, J. Wong-Leung, P. N. K. Deenapanray, H. H. Tan, C. Jagadish, B. Gong, R. N. Lamb, R. M. Cohen, W. Reichert, L. V. Dao, and M. Gal, J. Appl. Phys., 92, 3579 (2002).

15. P. N. K. Deenapanray, B. Gong, R. N. Lamb, A. Martin, L. Fu, H. H. Tan, and C. Jagadish, Appl. Phys. Lett., 80, 4351 (2002).

16. M. Buda, L. Fu, J. Hay, P. N. K. Deenapanray, H. H. Tan, C. Jagadish, P. Reece, and M. Gal, in Integrated Optoelectronics, M. J. Deen, D. Misra, and J. Ruzyllo Editors, PV 2002-4, the Electrochemical Society, Proceedings Series, Pennington, NJ (2002).

17. Y. M. Haddara, M. D. Deal, and J. C. Bravman, Appl. Phys. Lett., 68, 1939 (1996).

18. R. M. Cohen, Mater. Sci. Eng., R., 20, 167 (1997), and references therein.

19. N. H. Ky, L. Pavesi, D. Araújo, J. D. Ganière, and F. K. Reinhart, J. Appl. Phys., 69, 7585 (1991).

20. D. G. Deppe and N. Holonyak, Jr., J. Appl. Phys., 64, R93 (1989).

21. P. N. K. Deenapanray, L. Fu, M. Petravić, C. Jagadish, B. Gong, and R. N. Lamb, Surf. Interface Anal., 29, 754 (2000).

22. J. H. Teng, S. J. Chua, Y. H. Huang, G. Li, Z. H. Zhang, A. S. Helmy, and J. H. Marsh, J. Appl. Phys., 88, 3458 (2000).

23. A. Mitonneau, G. M. Martin, and A. Mircea, Electron. Lett., 13, 666 (1977).

24. D. Stievenard, X. Boddaert, and J. C. Bourgoin, Phys. Rev. B, 34, 4048 (1986).

25. R. Leon, P. Werner, K. M. Yu, M. Kaminska, and E. R. Weber, Appl. Phys. A: Mater. Sci. Process., 61, 7 (1995).

26. J. Lagowski, D. G. Lin, T.-P. Cehn, M. Skowronski, and H. C. Gatos, Appl. Phys. Lett., 47, 929 (1985).

27. P. N. K. Deenapanray, H. H. Tan, C. Jagadish, and F. D. Auret, Appl. Phys. Lett., 77, 696 (2000); J. Appl. Phys., 88, 5255 (2000).

28. P. N. K. Deenapanray, H. H. Tan, and C. Jagadish, Appl. Phys. A: Mater. Sci. Process., Online (2002)

29. P. N. K. Deenapanray, A. Martin, S. Doshi, H. H. Tan, and C. Jagadish, Appl. Phys. Lett., 81, 3573 (2002). 